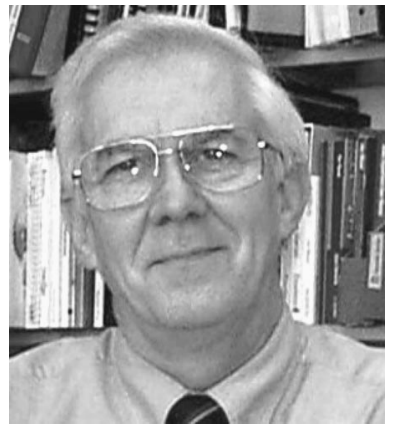

\section{Naming a New Society}

According to experts on mergers, one of the things that can sink a merger is the inability to reach agreement on the name of the new organization. In a takeover, it is not much of a problem. But in an amalgamation of equals, such as SPIE and OSA, it could be. The establishment of a name for a society signals to old and new members the directions and emphasis of the new organization.

Consider the dilemma of SPIE. Formed in the early days of aeronautical photography, the initials SPIE, the Society of Photographic Instrumentation Engineers, made sense to its members. But when the Society began to expand and include other fields of optical engineering in their conferences, the initials and name became confining. First the word, "photographic,', was changed to "photooptical" and later the name was extended with an explanatory title, "The International Society for Optical Engineering.',

The amended name does solve the problem of describing the direction of the society. But it is a problem for anyone in a leadership position who has to explain what SPIE stands for on a regular basis. Although SPIE does stand for the Society of Photo-Optical Instrumentation Engineers, explaining this and how it relates to an International Society for Optical Engineering can take a lot of time. We could have the same problem with the proposed new merged society.

What about the name "the Optical Society of America'"? Chosen when the Society was formed in 1916 , it is certainly clear what the organization is about, but the name does present some difficulties if it is to represent a global organization with no national biases.

When the Joint Task Force began to discuss what the new organization might look like, it became awkward to talk about a new organization, unless we had something to call it. Tony Seigman, an incredibly inventive gentleman, came up with the idea of calling the prototype organization "UNO" because the name is short and it gives the connotation of a single entity. One drawback to this choice was that "UN" in many minds stands for the United Nations and because this is an organization of op- tical societies, the " $\mathrm{O}$ " might be considered to stand for optics. So it might be construed as an acronym standing for the "United Nations of Optics." Certainly this was not the intention of the Task Force; using "UNO" was only as a matter of convenience. Some wag on the Task Force noticed that it might also be an acronym for "UnNamed Organization." The Joint Task Force tried to make it clear in any report it issued that the term "UNO", was a temporary term of convenience.

But why worry about the name of a new society before the members have even decided that they want OSA and SPIE to unify? It would seem that the questions to be answered first are: (a) whether to combine the two existing societies into a single organization; assuming the answer to (a) is "yes," (b) what should the organizational structure be; and (c) what are the bylaws for the organization that will assure the values of OSA and SPIE are preserved but that also allow for effective evolution of the unified organization. But the choice of a name is one of the top four reasons mergers fail! I can only conclude that by examining the choice of a name we might illuminate the criteria for reaching objective answers to the above three primary issues.

What should this projected new society be called? The Joint Task Force on the OSA/SPIE collaboration has danced around the issue. Paul Forman, the OSA co-chair, asked those on the Task Force to think of some possible names during our deliberations. He then scheduled a time at the end of one of the meetings to go over suggestions from the group. It was not pretty. Most of my colleagues produced a choo-choo train of letters that tried to encompass all the disciplines (optics, optical science, optical engineering, photonics, etc.), geographies (International, "of America"), and structures (society, institute, federation, organization, etc.) that one could possibly think of. None of them felt right. They didn't capture the spirit of this hybrid merger of operations and federation of cultures. And I think the name should do this.

Why do I care so much about this issue? Foremost, because it will be my society and names do count in the perception of others. But also, I have an abiding interest in graphic design and I know that a long string of letters defeats good design. For example, I think the recent redesign of the OSA logo and publication format to be ex- 
amples of excellent design. (Having said that, I still love Fig. 2 from Newton's Optiks that was used as OSA's logo for many years. So much optics is represented in that single diagram.) One possible strategy that would permit a fairly simple expression of the organization would be the "Society of Optical Science and Engineering.' And like the Society for Imaging Science and Technology, IS\&T, "Society" could be dropped from the initials to get "OS\&E" for use in a logo or as a shorthand acronym. The ampersand should be included both for clarity and for use as a graphic device.

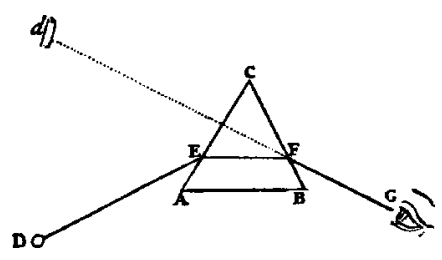

Fig. 2

I think that anything longer than that defeats the purpose of a name. Still, it doesn't do justice to our new organization. It's a bit mundane for a dynamic new society. After mulling over an alphabet soup of possibilities, I decided that no set of letters strung together from a structure and a set of disciplines, that would have to be both concise and inclusive, would be good enough for the organization. So I tried a different approach: find something that works. Of all the renamings of recent years, the most successful, to my mind, is that for the old AT\&T Bell Labs. When it was spun off from its parent organization it became Lucent. In an unabridged dictionary the definitions of the word are: 1. giving off light; shining. 2. translucent. Certainly, even without knowing the definition, the sound of the word connotes clarity and light. It also made people ask (and seek an answer), "What is that organization?' Is there a corresponding word or a near-word for our new society?
I ran some options by a guy in the SPIE leadership. He liked the concept of a name that was pronounceable, but that wasn't an acronym. For one thing he said it was slightly contrary to conventional practice, so people might sit up and take notice. But, as noted earlier, he also said that there was one big drawback: most society "names" are acronyms, so if the name doesn't stand for anything, explanations will be required. But given this from the outset, I still think it is the best approach as long as the name connotes or is evocative of optics, is new (for a brand new organization), it doesn't favor either of the current organizations, and it has no national connotations since the new organization must be truly international in character. I have developed my favorite new name, and even done some graphical design work. But I will save it for a future date after the society has received your input on the proposed unified organization.

In the meantime, evaluate all the opportunities and advantages of a unified SPIE and OSA (the report is available on line at www.spie.org/info/jtf/report/). For example, you could belong to the SPIE division of UNO (remember "UNO' is a placeholder for the final name) or the OSA division of UNO. The education efforts of the two societies are combined into the UNO Education Committee; all of the publications of the divisions would be published and accessible as part of the UNO Digital Library. Students could join UNO initially and then when their interests were better defined extend their membership to one of UNO's member societies or technical divisions. But organization of meetings would still be the precinct of the SPIE and OSA divisions of UNO (or of other member society or technical division that may exist in the future of UNO). At the beginning of this new year, I would like to wish all of our readers a most productive time. And that our societies will continue to progress toward unification. So here's to !!!

Donald C. O'Shea Editor 